\title{
Généalogie de la violence
}

La famille mise à nu dans El chico de la última fila de Juan Mayorga

Joana Sanchez

\section{(2) OpenEdition}

Journals

Édition électronique

URL : https://journals.openedition.org/cher/1893

DOI : $10.4000 /$ cher. 1893

ISSN : 2803-5992

Éditeur

Presses universitaires de Strasbourg

\section{Édition imprimée}

Date de publication : 1 décembre 2017

Pagination : 175-191

ISBN : 978-2-86820-962-7

ISSN : 1968-035X

Référence électronique

Joana Sanchez, "Généalogie de la violence », reCHERches [En ligne], 19 | 2017, mis en ligne le 01 décembre 2021, consulté le 11 février 2022. URL : http://journals.openedition.org/cher/1893; DOI : https://doi.org/10.4000/cher.1893

\section{(c) $)(1)(2)$}

Ce(tte) œuvre est mise à disposition selon les termes de la Licence Creative Commons Attribution Pas d'Utilisation Commerciale - Partage dans les Mêmes Conditions 4.0 International. 


\title{
Généalogie de la violence \\ La famille mise à nu dans El chico de la última fila de Juan Mayorga
}

\author{
JOANA SANCHEZ ${ }^{1}$
}

\begin{abstract}
No hay oficio más cruel que el de escritor, porque se expone, se desnuda y desnuda. Esa valentía, la de mirar algo de lo que los demás apartan la mirada, es el núcleo del talento mismo.

Juan Mayorga
\end{abstract}

$\mathrm{D}$ epuis quelques années, un thème est récurrent sur les planches du théâtre espagnol et latino-américain, celui de la «famille dysfonctionnelle» selon l'expression consacrée par la critique ${ }^{2}$ - devenue selon Begoña Donat «la protagonista del nuevo teatro" (2014). La vague dysfonctionnelle est lancée en 2005 quand l'argentin Claudio Tolcachir rencontre le succès que l'on sait avec sa pièce La omisión de la familia Colemán. Depuis, familles déchirées, éclatées, fragmentées, décomposées ou recomposées, occupent le devant de la scène théâtrale à travers des huis clos qui interrogent les mutations récentes du fait familial, mais reflètent aussi un certain effet de mode, comme un patron

1 Doctorante au sein de l'équipe d'accueil CHER (Culture et histoire dans l'espace roman) de l'Université de Strasbourg. Ancienne élève de l'École normale supérieure de Lyon, agrégée d'espagnol.

2 Si l'adjectif «dysfonctionnel» semble renvoyer à un certain usage biologisant ou psychanalytique du champ sémantique de la "dysfonction", l'expression «famille dysfonctionnelle» ne repose pourtant pas sur une solide assise conceptuelle et scientifique. Elle est avant tout le produit de la critique théâtrale et s'impose aujourd'hui avec la force de l'évidence dans la presse culturelle, voire dans certains écrits universitaires, faisait fi de son absence notoire de définition et de l'impensé qui règne sur les présupposés d'une telle étiquette catégorielle. 
dramatique quelque peu formaté et parfois vidé de sa substance, ce qui fait dire à Begoña Donat, non sans ironie: "Que empiece la disfunción» (2004). Or, à rebours de cette tendance et au moment même où elle prend son essor, Juan Mayorga, toujours soucieux d'éviter lieux communs et sujets éculés, s'empare également du thème familial, dans sa pièce El chico de la última fila, écrite pour la première fois en $2006^{3}$. Une fois de plus, Mayorga nage à contre-courant et cherche à écarter son regard des stéréotypes pour explorer la réalité familiale, non pas dans ces dysfonctions spectaculaires, mais dans le non-dit de son fonctionnement quotidien, pour sonder ses profondeurs inavouables et ses replis inattendus. Dans un jeu complexe d'enchâssement de récits et de théâtre dans le théâtre, la pièce raconte l'histoire d'une intrusion: celle de Claudio, un jeune garçon qui s'immisce dans la maison et la famille d'un de ses camarades de classe, Rafa. Ou plutôt c'est Claudio qui le raconte directement, à travers treize rédactions qu'il rend à son professeur de Lettres, Germán, qui les lit avidement, en compagnie de sa femme, Juana, formant ainsi une chaîne de réception complexe (et dont le dernier maillon est le spectateur), qui met en abîme le voyeurisme que suppose l'intrusion dans le cercle familial. Si la famille de Claudio semble être défavorisée et pourrait être taxée de «dysfonctionnelle» (son père est malade, ils vivent dans un quartier pauvre et sa mère les a abandonnés), celle-ci est soigneusement évitée par le dramaturge, contournée, escamotée à dessein et à peine évoquée, entre les lignes, par de maigres allusions périphériques, pour donner de l'épaisseur au personnage de Claudio ${ }^{4}$. Au contraire, la pièce met les projecteurs sur la famille de Rafa Artola, banale famille de classe moyenne absolument normale et "fonctionnelle», aimante, protectrice et apparemment libre de conflits; or c'est précisément cette «normalité » qui fait son intérêt pour Claudio - et à travers lui, mais pour des raisons bien distinctes, pour Mayorga comme il s'en explique dans sa seconde rédaction:

¿Por qué Rafa?, ¿por qué lo elegí a él? Porque él está en el extremo OPUESTO. Él es normal. Hay otros de la clase que están en el extremo opuesto, pero hubo algo, el curso pasado, que me hizo fijarme en Rafa: a menudo, al salir de la clase, vi a sus padres esperándolo, cogidos de la mano. A otros chicos les avergüenza que sus

3 Cependant, nous ferons référence, tout au long de notre travail, à sa dernière édition en 2014 au sein de l'ouvrage Teatro 1989-2014 (Mayorga 2014a).

4 On comprend entre les lignes de la première rédaction que Claudio vit dans un quartier pauvre quand il écrit subtilement «Yo sabía que si aceptaba, sería en su casa, porque la mía está en una calle que Rafa no pisará jamás» (Mayorga 2014a: 429). Plus loin, Claudio racontera à la mère de Rafa, pour l'émouvoir et gagner sa sympathie, que sa propre mère les a abandonnés son père et lui (2014a: 448) et, à la fin de la pièce, quand Rafa menace Claudio de se venger sur sa famille, il évoque une mystérieuse maladie de peau qu'aurait le père de son ami: "¿Qué le pasa a tu viejo en la piel?» (2014a: 468), mais Claudio ne répondra pas à la question. Trois allusions obliques donc, mais nous ne saurons rien de plus sur la famille de Claudio qui refuse d'en parler et la repousse sciemment dans le hors-scène et le hors-texte - «Mi padre no es un personaje de esta historia. Mi padre no sale» (2014a: 450) -, reflétant la stratégie d'évitement de la famille dysfonctionnelle du dramaturge. 
padres vayan por allí, porque les avergüenza la situación o porque se avergüenzan de sus padres. Rafa no. Rafa parecía conforme. Y yo me preguntaba: ¿cómo será su casa? ¿Cómo será la casa de una familia normal? (Mayorga 2014a: 433)

C'est ce déplacement du regard, de la famille problématique à une famille apparemment normale, qui, comme souvent chez Mayorga, ouvre une réflexion féconde sur la complexité des relations humaines. Or si la pièce est souvent étudiée à partir du rapport maître/ élève ou de la théâtralisation de l'écriture (Rodríguez Solás 2012), j'ai cherché à décentrer l'analyse de ces problématiques saillantes, à déplacer moi aussi mon regard, pour interroger le texte en changeant de perspective et en prenant la famille comme point d'appui. Comment le regard intrusif de Claudio dévoile-t-il peu à peu des failles, des fissures, dans le bonheur parfait de la famille Artola? Comment perçoit-on, entre les lignes, des frustrations, des rapports complexes de domination et de pouvoir, non seulement dans la maison de Rafa, mais également dans l'autre foyer visible sur scène, celui du couple intellectuel de Germán et Juana, à travers un habile jeu de parallélismes? Avec la pudeur et la limpidité tranchante qui lui sont propres, l'écriture de Mayorga débusque la violence dans la texture paisible du quotidien, met à nu l'espace domestique et éclaire subtilement cette remarque, apparemment provocatrice et cynique, de Germán: «¿No hay algo anormal, monstruoso incluso, en el concepto mismo de familia?» (2014a: 451).

\section{La déconstruction du fantasme de la famille idéale}

Claudio a choisi la famille de Rafa parce qu'elle était «normale» et cette «normalité» se cristallise à ses yeux dans la vision mythique à partir de laquelle tout commence: celle de Rafa avec ses parents se tenant la main à la sortie de l'école, image d'Épinal de la famille nucléaire, portrait de l'union familiale sacrée, qui éveille immédiatement désir et fascination chez un garçon dont la réalité domestique est toute autre, lui qui vient de ce que les sociologues du XIX ${ }^{e}$ siècle auraient appelé une famille «instable» (Le Play 1884), ceux du début du $\mathrm{xx}^{e}$ siècle une famille "brisée» (Durkheim 1897), et les critiques d'aujourd'hui - se faisant les relais improbables de la sociologie d'hier - une famille "dysfonctionnelle» (Donat 2014). Si la sociologie actuelle récuse ces terminologies (Eid 2008), c'est parce qu'elles sont toutes construites en référence à un modèle normatif unique, qui apparaît aujourd'hui comme un leurre: celui de la famille en tant qu'élément minimal de la société (Comte 1853), c’est-à-dire comme unité indivisible, communauté naturelle harmonieuse, modèle idéal de cohésion affective et sociale, comme le rappelle le philosophe Jean-Philippe Pierron:

On a l'habitude, sinon de penser, du moins de se représenter la famille en images. Celle de la cellule familiale, métaphore organique, est récurrente comme un reste romantique de la similitude. [...] Mais cette métaphore de la «cellule de base» a ceci de trompeur qu'elle exalte une unité dans la semblance en négligeant l'altérité qui tiraille la famille. (2014a: 141) 
Exalter "l'unité dans la semblance», cela veut dire considérer la famille comme une monade, comme un bloc indivisible, et nier l'existence de l'altérité au sein de celle-ci: c'est ne voir que le "nous", là où ce dernier est constitué de divers «je», ayant chacun des intérêts et des désirs qui peuvent différer. C’est passer sous silence les conflits familiaux et refuser de voir l'altérité dans celui qui est à côté de soi (son frère, son père, son fils) pour la renvoyer à celui d'en face: l'autre est rejeté hors du cercle familial, dans l'espace extérieur ou dans la figure liminale de l'intrus, que va endosser Claudio chez Mayorga. Si les sciences sociales s'accordent aujourd'hui à penser la famille en tant qu'espace de conflits, comme un «champ» (Bourdieu 1993) et un «dispositif» (Agamben 2006), le fantasme de la famille idéale hante toujours les représentations collectives, tel un horizon mythique inatteignable et culpabilisant, sans cesse exposé à nos yeux par les images publicitaires de familles tout sourire, glorifiant un bonheur sans tache dans la convivialité du traditionnel repas de famille, pour mieux nous vendre les produits non moins traditionnels de l'industrie agro-alimentaire.

Or ce mythe de la famille idéale repose sur une autre représentation profondément ancrée dans l'imaginaire collectif: celle de la distinction nette entre sphère publique et sphère privée, qui retranche la famille dans l'espace rassurant du foyer et voudrait en faire un isolat, un espace fermé, hermétique et douillet, l'espace du bonheur, du confort, du sweet home inventé par la bourgeoisie du XIX ${ }^{e}$ siècle et qu'on retrouve en filigrane chez Mayorga dans la passion de la mère de Rafa pour la décoration intérieure. À l'intérieur du cercle familial répond donc l'intérieur de la maison, et à l'altérité de celui qui n'est pas de la famille, l'altérité de l'espace extérieur, comme l'énonce clairement l'historien Roger-Henri Guerrand (1991: 173): «L'espace se répartit symboliquement en intérieur-famille-sécurité / extérieur-étranger-danger». À partir de là, s’instaure toute une dialectique autour de la dichotomie dedans/dehors qui peut se décliner selon plusieurs axes problématiques en famille / travail, femme / homme, solidarité/compétition, communion / conflit, calme / agitation, ami / ennemi, etc. Autant de polarisations que l'on retrouve dans la construction dramatique de la famille de Rafa chez Mayorga: la mère est au foyer alors que le père travaille; l'harmonie des relations familiales est symbolisée par la complicité père-fils au basket et la rhétorique du "Trabajo en equipo» vantée à plusieurs reprises par le père (Mayorga 2014a: 436) alors que ses relations professionnelles dans l'entreprise sont conflictuelles et ne cessent de se dégrader; la famille mange paisiblement tout en regardant les violentes émeutes de banlieues à la télévision (2014a: 454); etc. La liste n'est pas exhaustive. Mais si Mayorga semble souscrire, à première vue, à cette dialectique intérieur / extérieur, c'est pour mieux la faire voler en éclats grâce au personnage ambivalent de Claudio. Car ce-dernier est un intrus, c'est-à-dire quelqu'un dont la présence remet en question les limites du dedans et du dehors, qui est encore un étranger, comme le rappelle Ester, la mère: "No podemos sacrificar a Rafa por ayudar a un ajeno" (2014a: 445), mais qui est déjà à l'intérieur du sanctuaire. Plus précisément, au moment où Ester prononce ces mots, Claudio les écoute depuis le couloir, espace liminal 
par excellence, que Peter Von Matt (1998), dans son étude des écrits de Kafka, définit comme le territoire propre aux personnages problématiques situés sur les marges (mais pas en dehors) du cercle familial. L'intrusion est en soi un acte qui dynamite l'intimité paisible du huis clos familial, mais c'est surtout le regard intrusif - et par conséquent extérieur, distancié, ironique - de Claudio, à travers le portrait dressé dans les treize rédactions qu’il remet à Germán, qui va mettre à nu la famille Artola, la dépouiller de ses apparences lisses et sans conflit et lui ôter les oripeaux de la famille idéale.

Comme souvent chez Mayorga, la clef de ce levier dramatique est inscrite dans le texte même, à travers les paroles de Juana, la femme de Germán, qui dirige un magasin d'art contemporain et présente ses nouvelles acquisitions à son mari dans une tirade dont la portée métathéâtrale est évidente:

Son objetos normales, pero manipulados para producir un extrañamiento. Fíjate en el reloj: trece números. El artista interviene en el espacio doméstico poniendo de manifiesto rasgos que, de tanto verlos, ya no percibimos. Así consigue mostrar la mecanización de nuestra vida y desafiar las fronteras entre lo interior y lo exterior, entre lo privado y lo público. (2014a: 440-441)

Le regard de l'artiste est un regard décalé qui dévoile la porosité des limites entre le dedans et le dehors et l'inconsistance des représentations que celles-ci véhiculent. Le passage se poursuit avec la présentation par Juana d'un autre artiste contemporain qui fait de la "pintura verbal», c'est-à-dire qui enregistre une description de ses tableaux - treize aquarelles, faisant échos aux treize rédactions de Claudio -, dans un format radiophonique à mi-chemin entre la narration et le théâtre. Ces "peintures verbales» exigent, selon Juana, un rôle actif du spectateur et fonctionnent comme une métaphore de la pièce et de la dramaturgie de Mayorga ${ }^{5}$ :

Pintura verbal. Es la voz del pintor describiendo el cuadro. El espectador, o sea, el oyente imagina el cuadro. El espectador es un co-creador: vuelca su imaginario en la pared vacía. El artista propone que los auriculares cuelguen en una pared, o en un marco vacío. Para burlarse de la industria cultural obsesionada con la producción de objetos tangibles, él opta por intervenciones poéticas efímeras despojadas de materialidad. Las pinturas realmente existen, es decir, existieron, pero el pintor, después de hacer las descripciones en una grabadora, las destruyó. Trece acuarelas. (2014a: 441).

Derrière l'artiste dont parle Juana, il y a Claudio écrivain, utilisant le «cadre vide» de la page blanche et exigeant de ses lecteurs, Juana et Germán, une recomposition imaginaire de ce qu'il raconte, recréation qui prend corps dans

5 Il est manifeste que le passage marque aussi une distanciation ironique de la part de Mayorga vis-à-vis de certains usages abusifs du concept de "co-création" par le spectateur, dans l'art contemporain. Les «peintures verbales» décrites par Juana sont des caricatures qui confinent à l'absurde, mais il n'en reste pas moins que la réplique comprend des clefs de lectures pour l'ensemble de la pièce comme le révèle la correspondance - qui ne peut être fortuite - entre les treize rédactions et les treize pièces artistiques. 
la pièce quand les personnages du récit de Claudio apparaissent sur scène et «rejouent» la réalité racontée, ou «représentent» le récit. Mais l'artiste, c'est aussi Mayorga dramaturge, utilisant "l'espace vide» de la scène et exigeant des spectateurs un regard actif et co-créateur de l'œuvre. Il y a donc une mise en abyme claire du travail d'écrivain et, par conséquent, une mise à nu de la famille qui se fait à plusieurs niveaux.

Le premier niveau de dévoilement se fait par le regard de Claudio qui est un regard essentiellement ironique, et dresse un portrait de la famille Artola frôlant la parodie, comme le lui reproche Germán à plusieurs reprises. Claudio a beau répéter qu'il se contente de rapporter des faits bruts, son professeur, qui a l'expérience du poids des mots, sait bien que l'ambition de réalité en littérature est une illusion positiviste et que tout discours implique un point de vue, un agencement, un processus de sélections, des silences, des ellipses, c'està-dire une mise en ordre dont l'écrivain est souverain et dont dépendent les significations du texte ${ }^{6}$. Claudio construit ainsi un récit elliptique qui escamote les liens de causalité et rend les situations absurdes et les personnages risibles, comme on le voit très clairement dans la troisième rédaction quand le père de Rafa lui propose de rester dîner pour voir un match :

Acepto la oferta del hombre del chándal. Una hora después nos reunimos con él en el salón, aunque yo tardo en reconocerlo, sin chándal parece otra persona, pero por cómo se relaciona con el mando de la tele deduzco que sí, es él, el cabeza de familia. Está interesado en que los Grizzlies ganen a los Clippers. En los Clippers juega un coreano, lo que le da pie a hablarnos sobre China. En el tercer cuarto se incorpora la madre, a ver el partido o a informarse sobre China. (2014a: 437)

Les personnages sont réduits à des apparences n'ayant d'identité que par leur costume ou leurs rôles familiaux. Le point de vue extérieur et fragmentaire de Claudio rend les enchaînements absurdes et l'allusion à la télécommande comme sceptre de l'autorité patriarcale vient couronner l'ironie du texte. Dans le premier montage de la pièce par la compagnie Ur Teatro, en 2006, les scènes dans la maison de Rafa étaient d'ailleurs marquées par un jeu caricatural et outré soulignant ce point de vue déformant de Claudio, qui n'échappera ni au lecteur ni au spectateur. Mais c'est à un deuxième niveau, entre les lignes du texte de Claudio, au-delà de son regard et de sa conscience, que se situe le véritable

6 «Germán- [...] Pero si lo que tú quieres es ser un caricaturista... ¿Es eso lo que quieres ser, un caricaturista?

Claudio - Usted dijo que los mirase de cerca. Cuanto más de cerca los miro es peor. Escribo lo que veo» (Mayorga 2014a: 440).

L'équivoque repose, me semble-t-il, sur la mauvaise compréhension par Claudio de l'injonction du professeur à "regarder de près » : alors qu'il l'invitait à avoir un regard "hospitalier» - pour reprendre un terme cher à Mayorga -, compréhensif, à ne pas regarder depuis les marges avec la distance du point de vue extérieur, mais à chercher à adopter le point de vue des personnages, Claudio a pris la remarque au sens premier et fait du "regarder de près», un regard grossissant et par conséquent forcément déformant et caricatural. 
dynamitage de la famille idéale, dont le réel artisan est le dramaturge, ainsi que le lecteur attentif capable de repérer ces fissures dans les non-dits du texte. Prenons par exemple le moment où Claudio observe une photo de famille dans le salon des Artola:

Sobre la tele, junto a un dragoncito chino, foto de la sagrada familia en la playa, de cuando Rafa era pequeño: papá, mamá, el nene y una nena algo mayor que Rafa. El dragón los miraba como si fuera a devorarlos todos. (2014a: 434)

L'évocation du dragon chinois dévorateur jette le trouble sur l'image d'Épinal de la famille réunie et laisse planer un malaise par association d'idées. Par ailleurs on est surpris de l'évocation de "una nena», la sœur de Rafa, qui est la grande absente de la pièce et de la maison. Jamais présentée, à peine évoquée au détour d'une allusion anodine, on ne parlera jamais d'elle, mais son absence est étrangement présente et résonne à plusieurs reprises entre les lignes du texte, comme une fissure dans la cohésion familiale, une béance qui se fait jour là où la famille ne semblait être que plénitude. C'est le cas par exemple quand Claudio, qui fouille les tiroirs de la maison, trouve des cartes postales d'elle et écrit simplement "la última de hace tres años» (2014a: 457), où quand on le fait dormir dans la chambre de l'absente "que ahora es el cuarto de la plancha» (2014a: 463). On comprend que Marta ne vient plus, et la description de sa chambre ornée de poupées mutilées, vestiges d'un temps révolu, est à la fois macabre et dérangeante: "Es el cuarto de una niña de catorce años, aunque Marta debe de tener unos veinte. Hay una estantería llena de Barbies. Pero si están... Una manca, otra tuerta... Están todas mutiladas, pobres» (2014a: 464). La mutilation est une manière d'inscrire l'absence dans le corps et on peut voir dans ces poupées lugubres une projection de la présence fantomatique de Marta dans la maison. Mais le personnage de la sœur n'est pas la seule figure spectrale dans cette maison: Ester, la mère, est à la fois omniprésente et comme absente à elle-même: «La señora se quedó en el salón, con la revista en la mano y el metro en la otra, flotando como un fantasma», écrit Claudio juste après avoir évoqué la photo de famille sur la télévision (2014a: 434). C'est un personnage apathique, constamment dans une présence-absence liminale, comme dépossédée d'ellemême, prisonnière de rêves chimériques. Pourtant, ses rêves semblent bien modestes: rénover la maison, changer la décoration... mais il faut y lire le besoin d'échapper au réel, d'abattre symboliquement les murs de la maison qui n'est plus un refuge, mais un carcan aliénant:

En el minuto cuatro, Ester se sienta a mirar el partido, pero no consigue interesarse, abre el número 215 de Casa y Jardín, por sus ojos desfilan una mansión de estilo victoriano, $[\ldots]$ tantas casas que ella no tendrá jamás. En el minuto siete, saca un lápiz y hace un boceto de reforma de la casa, tiene una carpeta llena de bocetos, se pasa el día en casa, pero no está conforme con la casa, está decidida a reformar la casa aunque sabe lo duro que es tener obreros en casa. (2014a: 439)

Ester, la petite bourgeoise aux petits rêves médiocres de décoration (méprisée pour cela par Germán qui ne voit que la parodie de la classe moyenne et le premier niveau de lecture) est pourtant une figure dramatiquement bovaryste 
qui incarne une insatisfaction, un mal-être et une vulnérabilité que l'on découvre peu à peu, entre les lignes, dans tous les personnages de la pièce et qui est la matrice de l'apparition de la violence chez des individus a priori «normaux", comme je l'analyserai plus en avant. Pour en revenir au personnage d'Ester, Claudio découvrira en fouillant dans la chambre à coucher qu'elle prend des anxiolytiques, tandis que le lecteur attentif saura voir aussi les allusions anodines mais répétées à l'alcool («Ester vuelve al salón con dos martinis» (2014a: 444), "Ella toma un martini» (2014a: 448), «Se sirve un martini» (2014a: 467), etc.). Si je me suis attardée sur le personnage de la mère, c'est parce qu'elle cristallise l'image de la famille idéale, désirée ardemment par Claudio (et le personnage d'Ester exerce aussi une attraction sexuelle sur le jeune homme), mais que l'on découvre brisée de l'intérieur, fantasmatique et chimérique. Ainsi, en pénétrant dans la maison, le regard de l'intrus met à nu ces faiblesses, dévoile l'envers du décor familial et démonte le fantasme de la famille idéale.

\section{Image dialectique et dramaturgie «liquide»}

Mais la déconstruction du mythe de la famille n'est pas une fin en soi et pour en comprendre les enjeux profonds, il faut sortir de la maison des Artola et mettre les scènes que l'on vient d'analyser en relation avec les autres espaces scéniques de la pièce: l'école, la maison de Juana et Germán, la boutique d'art contemporain. Car l'intrusion et l'effacement des frontières entre le dedans et le dehors deviennent aussi un principe de construction dramaturgique et permettent de tisser un réseau de sens à partir de pôles a priori antagoniques.

Pour ouvrir un champ de problématiques fécond, Mayorga construit souvent son théâtre à partir de ce qu'Erwan Burel appelle une «dramaturgie elliptique » ${ }^{7}$, concept qui s'enracine dans l'analyse que fait le dramaturge de l'ellipse chez Walter Benjamin (Mayorga 2010). Une ellipse est une figure géométrique qui fait la jonction entre deux points éloignés, non pas par une droite, mais à partir de deux «foyers » situés chacun à égale distance des deux points, ce qui crée un espace de tensions. Sans entrer plus en avant dans les subtilités mathématiques, l'idée essentielle du concept d'ellipse est la création d'une "image dialectique», d'un croisement sémantique à partir d'éléments contrastés, qui forme une zone ouverte à la pluralité du sens (par opposition à l'univocité de la droite, pour filer la métaphore géométrique):

Lo decisivo es que ninguno de los objetos sea luego pensado sin atender al otro y que el vínculo entre ambos haga aparecer un lugar que ninguno de ellos crearía

7 Les travaux d'Erwan Burel, encore inédits, concernant cette question sont les suivants: "Théâtralité elliptique et récupération de la mémoire historique dans Himmelweg et Le Cartographe de Juan Mayorga», lors du colloque Théâtralité(s) Orient/Occident à l'Université de Strasbourg les 15, 16 et 17 octobre 2014; «El escenario teatral como espacio elíptico: reflexiones acerca de la elipse en el teatro de Juan Mayorga », lors du congrès de la AIH (Asociación internacional de hispanistas) à l'Université de Münster du 11 au 16 juillet 2016 . 
por sí solo. [...] no es el vínculo entre dos objetos distantes, sino el lugar tenso y denso creado por un emparejamiento improbable. (Mayorga 2010)

Or El chico de la última fila est une pièce profondément elliptique du fait de l'existence de deux niveaux de fiction: la fable cadre, celle de la lecture et du commentaire des rédactions dont les personnages sont Claudio, Germán et Juana, et la fable enchâssée, le contenu des rédactions, c'est-à-dire le récit de ce qui se passe dans la maison des Artola. Aux deux pôles de la pièce, nous avons donc deux familles, deux espaces domestiques, qui a priori ne se croisent pas: la maison des Artola et l'appartement de Juana et Germán. Ces deux espaces sont par ailleurs prolongés chacun par un espace professionnel, dans le horsscène, l'entreprise du père de Rafa d'un côté, la boutique de Juana de l'autre (même si ce dernier espace s'actualise sur la scène à la fin de la pièce). Entre les deux pôles, un espace fait la jonction entre les deux ménages, l'école, troisième espace public, ainsi que les deux personnages qui y sont protagonistes, Claudio l'élève et Germán le professeur. Se dessine ainsi une ellipse dont les points A et $B$ seraient les deux groupes familiaux qui ne se rencontrent jamais directement, mais sont mis en tension sémantiquement par les deux personnages «foyers" de l'ellipse, Claudio et Germán. Et si l'on reprend la dialectique dedans/ dehors, ouvert/fermé, qui guide notre réflexion depuis le début, on peut dire que la structure elliptique ouvre, par un jeu dialectique de croisements et de prolongements, les deux espaces domestiques a priori fermés, et perfore les cercles familiaux à priori hermétiques. Au niveau de la fable, cela se traduit par une série de parallélismes permanents entre les deux familles, dont je ne citerai que quelques exemples: alors que le père de Rafa veut lancer une affaire d'import/ export avec la Chine, la femme de Germán expose des artistes d'origine chinoise; quand Germán répète - au sens théâtral - les excuses qu'il devrait faire à Rafa, Ester répète le discours de rupture qu'elle aimerait tenir à son mari (dans les deux cas, l'action sera d'ailleurs avortée); quand Claudio observe Ester endormie, il écrit qu'elle a la peau très blanche et de petits pieds (Mayorga 2014a: 464), ce qui entre en résonnance avec son observation de Juana endormie à la toute fin de la pièce, quand il réussit enfin à pénétrer dans l'autre maison et qu'il note qu'elle "a les pieds très blancs» (2014a: 472). On pourrait continuer la liste de ces micro-parallélismes qui criblent le texte d'échos et en font une vaste caisse de résonnance qui entrecroise les fables des deux niveaux de fiction et tisse subtilement une image dialectique. Dans sa mise en scène de la pièce, Jorge Lavelli rend admirablement bien ce jeu de renvois réfléchissant en plaçant au centre du plateau de grands panneaux miroirs qui se font face et que les acteurs vont déplacer (Gap 2009). Même l'intrusion de Claudio dans la maison est spéculaire, car quand Germán rapporte chez lui les rédactions et les lit avec sa femme, il permet aussi l'intrusion de son élève dans l'intimité de son propre foyer, intrusion par l'écriture, mais qui va peu à peu prendre corps du fait de l'effacement progressif des limites entre les deux niveaux de fiction.

On ne se situe plus ici au niveau de la fable, mais au niveau de la trame, de l'agencement des actions. Alors que dans Hamelin par exemple, les deux 
niveaux de fiction sont clairement distingués grâce au personnage de l'Acotador qui prend en charge le récit enchâssé, le principe structurant de El chico de la última fila est justement d'estomper les transitions d'un niveau à l'autre, d'effacer les limites du dedans et du dehors des rédactions et de se construire dans un va-et-vient périlleux et sans cesse mouvant entre le drame et le récit, la fiction enchâssante et la fiction enchâssée. Cet effacement est progressif: la première rédaction de Claudio est lue par Germán à haute voix si bien qu'elle s'insère parfaitement dans le dispositif mimétique du récit enchâssant. La seconde rédaction est récitée directement par Claudio, alors qu'on voit Juana et Germán lire, ce qui opère un dédoublement de la fiction et de l'espace scénique, et une véritable intrusion de la voix de Claudio dans la scène. Dès la troisième rédaction, Claudio ne récupère plus seulement sa voix, mais aussi son corps, et «rejoue» la scène racontée, accompagné des personnages de son récit qui s'incarnent sur scène. La distinction drame/récit, passage mimétique/passage épique, ne permet donc plus, comme au début, de distinguer les deux niveaux de fiction et on a une alternance complexe entre scènes enchâssantes représentées, récits enchâssés assumés par Claudio et scènes enchâssées représentées comme dans un flash-back. À cela il faut ajouter que les scènes enchâssantes sont ellesmêmes dédoublées entre les commentaires que Juana et Germán font chez eux et ceux que Germán fait à Claudio à l'école, alors que très souvent aucune indication scénique ne signale le passage d'un niveau à un autre. La dramaturgie repose donc sur ce jeu d'enchevêtrement du temps, de l'espace et des situations, dans un vertigineux exercice d'équilibriste qu'illustre bien l'exemple suivant:

GERMÁn - [...] Saca de la cartera una redacción. ¿Quieres leerlo?

Juana no contesta, pero se acaba acercando a leer.

RAFA - (A Claudio, leyendo sus apuntes) «A tu padre le han puesto una multa de tráfico. Él considera que es injusta y se plantea no pagarla. ¿Qué le aconsejaría Sócrates?»

GERMÁn - ¿Qué démonios es esto?

Claudio - El de Filosofía está empeñado en convencernos de que su asignatura es útil. Siempre empieza planteándonos un caso, él lo llama «dilema moral», y luego nos explica el filósofo; Platón, Hegel, lo que toque. Todos quieren convencernos de que enseñan cosas útiles. Todos menos el de Matemáticas. Ése ya nos advirtió el primer día que las Matemáticas no sirven para nada.

Germán - Las Matemáticas son importantes. También la Filosofía. Aunque ni las Matemáticas ni la Filosofía tengan respuesta para la gran pregunta.

Claudio - ¿La gran pregunta?

(Mayorga 2014a: 441-442)

La première réplique se situe dans l'espace domestique de Juana et Germán qui se mettent à lire ensemble, ce qui produit un "saut» à l'intérieur du récit de Claudio où Rafa personnage «rejoue» ce qui est raconté dans la seconde réplique. Mais Germán réagit immédiatement au récit et nous propulse de nouveau, par son commentaire, hors du récit enchâssé. Néanmoins, on ne sait plus s'il s'adresse à Juana pour commenter ce qu'il lit ou s'il s'adresse à Claudio à l'école, dans une soudaine prolepse, comme le laisse penser la dernière réplique 
de Claudio qui lui répond directement. Quant à la réplique centrale de Claudio, impossible de savoir s'il s'agit d'un commentaire du narrateur intégré à la rédaction ou d'une réponse qu'il fait directement à Germán. Prolepses, analepses, saut temporel, saut spatial, récit enchâssant, récit enchâssé, Mayorga malmène le cadre spatio-temporel classique et nous offre une trame bousculée au point que Davide Carnevali parle de "colapso del microcosmos dramático» (2015: 168), car la trame se présente comme un épisode unique, comme un flux dramatique, tout en contenant une multiplicité de situations, d'espaces et de temporalités ${ }^{8}$. Mais la dramaturgie n'est pas seulement éclatée entre plusieurs niveaux de fiction: elle est poreuse, du fait de la fluidité mouvante de la trame, de ses situations perméables débordant les unes sur les autres, si bien que l'on pourrait parler d'une «dramaturgie liquide». La scène est transpercée de situations multiples qui s'infiltrent les unes dans les autres, dans une dynamique qui n'est pas sans rappeler l'intrusion de Claudio dans la maison". Grâce à ce dispositif «liquide» et intrusif, la pièce devient un espace ouvert, liquéfié, aux antipodes d'un huis clos familial, et joue subtilement de la dialectique dedans / dehors, privé / public, en rendant toute limite poreuse et perméable. Une fois de plus, la mise en scène de Lavelli sert magistralement le texte de Mayorga, à travers une scénographie dépouillée, neutre, où l'espace, toujours ouvert et mouvant, est découpé par les personnages sur scène et où «un jeu d'acteur chorégraphique» (Gap 2009) souligne la fluidité presque onirique de la dramaturgie.

\section{Humiliation et frustration: une généalogie de la violence dans les replis du quotidien}

$\mathrm{Au}$-delà du tour de force que constitue ce véritable ballet d'équilibriste, comment peut-on comprendre et interpréter cette dramaturgie audacieuse? Notre hypothèse est que la clé de voûte de toute la pièce est le dépassement des lignes de fracture intérieur/extérieur, fermé/ouvert, famille/travail et ami/ennemi exposées en première partie. La dramaturgie liquide abolit ces cloisonnements factices pour mettre à nu la complexité des relations humaines qui reposent toutes, quelle que soit leur nature, sur une tension complexe entre vulnérabilité et désir de domination. Les mêmes logiques de pouvoir traversent ainsi le couple de Juana et Germán, la famille Artola, mais aussi les rapports de chacun d'eux au monde professionnel, nous rappelant que la sphère privée n'est

8 «Como el tiempo, también el espacio es un flujo continuo, que no conoce los límites que requerirían las diferentes ambientaciones en que los acontecimientos tienen lugar. Así que los personajes no están obligados a pasar de una escena a otra, ni siquiera a entrar y salir: basta con nombrarlos para que aparezcan al instante. Ellos, al fin y al cabo, "siempre están ahí", en el espacio neutro del teatro. [...] el tiempo experimenta una aceleración que comprime la "fábula", creando puntos de conexión inusuales entre un acontecimiento y el sucesivo» (Carnevali 2015: 172-173).

9 Le bloc narratif de la rédaction est intrusif dans la fiction enchâssante, mais les commentaires de Germán sont aussi intrusifs dans la fiction enchâssée. 
pas un îlot de cohésion parfaite, mais se trouve infiltrée par les dynamiques de la sphère publique. La porosité de la dramaturgie est ainsi à l'image de la porosité des liens familiaux et sociaux et invite à repenser notre conception de l'extérieur et de l'altérité. Car la violence n'est pas l'apanage de l'autre, elle ne se situe pas seulement en face de soi, à l'extérieur (de soi, de sa famille, de sa culture, etc.), la réalité n'est pas si simplement binaire avec les bons d'un côté et les méchants de l'autre comme dans un film hollywoodien ou, pour le dire avec une terminologie malheureusement toujours prégnante dans l'espace médiatique contemporain dès que l'on parle de violence, il n'y a pas d'un côté la civilisation et de l'autre la barbarie. C'est un thème cher à Juan Mayorga, sur lequel il revient à de nombreuses reprises au fil de sa production, que ce dévoilement de ce qu'il appelle «esa condición paradójica del ser humano» (2014b), entre fragilité intrinsèque et désir de puissance et de domination, qui se fait jour dans des événements exceptionnels d'une extrême violence (comme on le voit avec la Shoah dans Himmelweg), mais aussi dans les replis silencieux du quotidien. El chico de la última fila met en lumière cette "zone grise» dans les milieux que l'on penserait les plus exempts de violence - l'école et la famille, les deux piliers de la «civilisation» dans l'idéologie dominante - et y découvre les germes de la barbarie et la matrice de la violence:

En lo que al teatro se refiere, la zona gris es la zona más rica. Los personajes que habitan en la zona gris, aquellos que siendo víctimas se convierten en algún momento en cómplices del verdugo, son figuras que yo he visitado con frecuencia en mi teatro. Como ciudadano, siento que en la medida en que consentimos injusticias, que aceptamos formas de barbarie, o de acoso del hombre por el hombre, estamos entrando en la zona gris. (Mayorga 2016)

Mayorga construit et dévoile en même temps cette zone grise chez chacun des personnages de la pièce. Commençons par le personnage de Claudio, le plus ouvertement ambivalent, qui se présente comme le grand orchestrateur de l'œuvre, celui qui réussit à s'introduire chez les Artola, à gagner leur sympathie tout en les méprisant, celui qui expérimente la puissance des mots à la fois pour convaincre et manipuler (notamment avec Ester et la scène du poème où il réussit à l'émouvoir aux larmes), mais aussi pour écrire et réécrire l'histoire à partir de son point de vue souverain. Claudio l'intrus, l'insensible, le garçon dérangé10, est aussi un adolescent vulnérable, touchant, et constamment sujet à des microhumiliations. On les perçoit, par exemple, dans le fait que les parents de Rafa ne retiennent jamais son nom et l'appellent inlassablement «Carlos", ce qui montre un désintérêt criant pour sa personne. C'est ce qu'on appelle en espagnol du "ninguneo", terme de psychologie n'ayant pas d'exact équivalent en français et traduisant cette relégation de l'autre qui peut être vécue comme une forme de

10 En Argentine, où la pièce a eu un franc succès, notamment auprès des psychiatres et psychanalystes, l'expression "chico de la última fila» est devenue un syndrome clinique désignant «un type d'adolescent issu d'une famille disloquée, agressif, manipulateur, mais doué, qui est à la recherche d'un père et d'une mère» (Le Run 2015). 
négation de son être et d'humiliation (Espeche 2014)11. De la même manière, quand Ester dit à son mari qu'il faudrait peut-être prendre un vrai professeur de mathématiques et faire sortir "cet étranger» de la maison, elle le fait en sachant que Claudio entend tout, comme s'il n'existait pas. Cette violence symbolique est si forte que Juana interrompt la lecture de la rédaction pour le faire remarquer à son mari. C'est cette vulnérabilité du personnage que Mayorga met en exergue dans le titre de la pièce El chico de la última fila: certes, cela renvoie à sa position d'observateur et, par enchâssement, à la position du spectateur, mais c'est aussi une manière de souligner sa position marginale, à la limite du dehors, que ce soit dans la classe, dans la maison de Rafa ou dans la société. "Se sienta en la última fila. No habla. No crea problemas. En las demás asignaturas no destaca ni por arriba ni por abajo», dit Germán (Mayorga 2014a: 435). Autrement dit, c'est un élève sans relief, quasiment invisible, "ninguneado». Cependant il va peu à peu s'affirmer et éprouver sa propre puissance, grâce à l'écriture, mais au risque d'entrer dans cette "zone grise» où l'oppressé devient l'oppresseur. Néanmoins, l'écriture fonctionne comme un exutoire, un garde-fou qui le retient, d'une certaine manière, d'exercer une violence physique directe, comme le comprend bien Germán: «Es un chico cabreado, sólo eso. Un chico enfadado con el mundo. Y no es para menos. Mejor que saque su rabia así y no quemando coches. A mí me dan miedo los otros» (2014a: 434). Si l'on s'attache à l'autre personnage central de la pièce, Germán, dont la femme dit à plusieurs reprises qu'il ressemblait beaucoup à Claudio quand il était jeune, on retrouve la même ambivalence. Quand la pièce s'ouvre, on découvre un professeur désabusé, éreinté, frustré, sans illusion, "humilié» (selon ses propres mots) par ses élèves au théâtre (2014a: 435). Mais, en même temps, on remarque, dès les premières répliques de la pièce, une dissymétrie frappante dans son rapport intime avec sa femme. Il monopolise quasiment la parole (avec de longs monologues) alors qu'elle a du mal à s'exprimer ou n'est pas écoutée. Il ne s'intéresse ni à ce qu'elle fait, ni à ses états d'âme et méprise ses activités professionnelles ${ }^{12}$. Autrement dit, dans le champ qu'est la famille et, ici plus précisément, le couple, Germán exerce une position clairement dominante et «ningunea» son épouse, comme lui se sent «ninguneado» en tant que professeur. Quand Claudio rencontre finalement Juana, il perçoit immédiatement cette faiblesse, cette humiliation silencieuse - la même qu'il avait perçue chez Ester - et la pointe du doigt lorsqu'il écrit:

11 "Los argentinos inventamos el término "ningunear", una variante de la indiferencia que descalifica el ser mismo de la persona, a quien se le niega existencia o significación, dejándolo en el limbo de la nada misma» (Espeche 2014).

12 «Mi mujer vende este tipo de cosas en su tienda, El Laberinto del Minotauro, o sea, un lugar para extraviarse. La han heredado dos señoras con sentido común, dos que llaman al pan, pan y al vino, vino, y le han dicho que se deje de arte para enfermos o le cierran el chiringuito» dit Germán, dans une réplique parmi de nombreuses autres où il méprise ouvertement le métier de sa femme. (Mayorga 2014a: 461). 
Siento que sabe mucho de mí, y yo casi nada de ella. Bueno, algo sé. Sé con qué clase de hombre está casada. Sé que no tiene hijos. Sé lo que piensa su marido de todo esto que la rodea: «mierda», «arte para enfermos». (2014a: 471)

Comme toujours, la violence est inscrite entre les lignes, dans les non-dits du texte et du couple. Dans la famille Artola, on retrouve le même rapport de force autour de la question du genre dans le couple parental. Parce que le père veut monter sa propre entreprise, Ester accepte de renoncer à reprendre ses études, parce qu'il faut investir dans ce projet qui n'est pas le sien, elle fait une croix sur ses rêves de transformation de la maison: c'est un personnage constamment en retrait, qui se sacrifie pour les autres, renie ses ambitions et sa personne. Mais elle endosse également le costume de l'oppresseur dans son rapport aux domestiques: avec «la mujer de piel oscura» (2014a:433) d'abord et on voit ici comment perce le rapport de classe à travers l'allusion à la couleur de peau - qu'elle réprimande pour avoir osé porter un de ses vêtements dont elle allait de toute façon se débarrasser, puis avec Luba dont Claudio écrit que son éducation dans le bloc de l'Est lui avait appris à savoir se faire invisible, et enfin avec les ouvriers roumains qu'elle envisage de faire travailler illégalement pour rénover sa maison (2014a: 444). Quant au père de Rafa, humilié au travail par son patron qui le tance devant toute l'entreprise pour n'avoir pas payé une facture, il envisage pourtant lui-même de créer une entreprise d'import-export avec la Chine parce que là-bas on peut fabriquer des contrefaçons pour trois euros seulement, en oubliant ce que cela implique au niveau humain: "Es como tener una fábrica sin obreros. O más fácil aún», dit-il (2014a: 443). Le rapport oppressé/ oppresseur devient complètement réversible. La domination devient le revers de l'humiliation, et la violence celui de la frustration. Le seul personnage qui semble libre de conflits dans un premier temps est Rafa, mais, pour donner de la matière au récit de son élève et de l'épaisseur à Rafa, Germán - se faisant complice de Claudio - va humilier publiquement Rafa en classe: «Fue como sentirme en pelotas. Nunca me había sentido tan humillado ", raconte ce dernier à Claudio (2014a: 455). Des stigmates de cette humiliation vont naître chez Rafa un désir de vengeance, une rage silencieuse, et une volonté de puissance dont une des issues est la violence: "Me gustaría que se sintiese como yo me sentí. Le daba de hostias y le quemaba el coche» (2014a: 455). Heureusement pour Rafa, il trouvera comme Claudio un exutoire dans l'écriture à travers l'article intitulé "La pizarra vacía», qu'il publie pour dénoncer l'attitude de son professeur. Son père, lui, ne trouvera pas d'autre issue que la violence: « $\mathrm{Ha}$ vuelto a sacarme la factura del chino, delante de todo el mundo. He estado en un tris de tirárselo a la cara, los trescientos euros. [...] Le he quemado el coche", raconte celui-ci à sa femme (2014a: 470). Cette violence soudaine semble absurde, irrationnelle, inexplicable, chez un bon père de famille, cadre dans une entreprise, un homme tout ce qu'il y a de plus normal, qui n'a rien à voir avec ces jeunes Français de banlieues que les personnages de la pièce regardent brûler 
des voitures à la télévision ${ }^{13}$. Mais c'est justement là que se situe le cœur de la réflexion de Mayorga. La matrice de la violence n'est pas le fait d'une inculture ou de la barbarie, mais le produit d'une tension entre humiliation et volonté de puissance chez des individus qui ne trouvent pas d'autre issue que l'agressivité, comme le comprend très bien le père de Rafa lui-même quand il commente les images télévisées des émeutes de banlieues: "Esos chicos no tienen horizontes. Les han cerrado todas las puertas. Así expresan su rabia contra un sistema que los excluye» (2014a: 454). C'est par ce croisement dialectique entre l'histoire française récente et la petite histoire des personnages que la pièce devient hautement politique et que la mise à nu des rapports humains devient aussi une radiographie sociale, proposant une autre généalogie de la violence que celle établie par le discours médiatique hégémonique. Mayorga construit des personnages ordinaires, d'une condition privilégiée même, et il dévoile ce qu'en psychanalyse on appelle leurs "zones prohibées", leurs frustrations, leur insatisfaction, leur sentiment d'impuissance et de défaillance dans une société dominée par une injonction à la réussite et au bonheur, comme le révèlent les lectures des parents Artola: «Ella, "La fórmula de la felicidad: aprende a ser tu mejor amiga”. Él, “¿Quien se ha llevado mi queso? Cómo adaptarse a un mundo en constante cambio"»(2014a: 457). Ces parodies de livres de développement personnel, rangées à côté des antidépresseurs sur la table de nuit, sont symptomatiques de la frustration endémique que produit une société qui exige que l'on soit ambitieux, que l'on colle à l'image du «winner» (Benasayag 2015), ou, pour reprendre une expression du père de Rafa, qu'on ait toujours envie de «dévorer le monde» (2014a: 444). Face à cet horizon de réussite sociale, les personnages de la pièce sont tous des «ratés", des individus qui souffrent de ce décalage entre leur situation et leurs ambitions chimériques, si bien que Juan Mayorga les compare à des «Don Quichotte» modernes (Sadowska Guillón).

Or, dans le même entretien (Sadowska Guillón), Mayorga définit sa pièce comme un "Bildungsdrama postmoderne», un "drame d'apprentissage à l'envers». Comment comprendre cette assertion? Le drame et le roman d'apprentissage commencent toujours par une sortie de la maison: le héros s'élance vers l'extérieur et affronte les dangers qui se dressent sur sa route. Dans El chico de la última fila, la dynamique est contraire: il y a intrusion à l'intérieur de la maison et parallèlement dévoilement introspectif des personnages qui découvrent petit à petit que la violence n'est pas seulement dehors, chez les autres, mais potentiellement en chacun d'eux, en chacun de nous. Et c'est paradoxalement chez les personnages adultes, censément mûrs et raisonnables, que cela éclate avec le plus de clarté: chez le père de Rafa, comme nous l'avons vu, mais aussi chez Germán, lui qui n’hésite pas, au début de la pièce, à qualifier

13 L'épisode des violentes émeutes de banlieues en France a lieu en 2005, peu avant l'écriture de cette pièce par Mayorga, et c'est une référence qui revient à plusieurs reprises dans le texte, que ce soit à la télévision, dans la bouche de Germán ou dans celle du père de Rafa. 
ses élèves de «barbares» en les méprisant ouvertement et en les rejetant dans une insurmontable altérité face à lui, intellectuel, cultivé, "civilisé»:

Elegí esta profesión pensando que viviría en contacto con los grandes libros. Sólo estoy en contacto con el horror. [...] Lo peor es imaginar el día de mañana. Esos chicos son el futuro. ¿Quién puede conocerlos y no hundirse en la desesperación? Los catastrofistas pronostican la invasión de los bárbaros y yo digo: ya están aquí; los bárbaros ya está aquí, en nuestras aulas. (2014a: 428).

La grande leçon que va apprendre Germán, de la main de son élève, dans ce drame d'apprentissage à l'envers, c'est que la barbarie ne se trouve pas seulement en classe, en face de lui, mais aussi en lui, comme en témoigne son basculement progressif dans la zone grise quand il se fait complice de Claudio, d'abord en volant une copie, puis en humiliant Rafa, et surtout quand il gifle son élève à la fin de la pièce. Cette gifle, la seule violence physique qui apparaît sur scène sans être escamotée dans une ellipse, est le point d'aboutissement de cet apprentissage, le moment où la violence souterraine éclate en plein jour chez le personnage symbolique du maître, de l'homme cultivé, qui ne peut alors plus fuir ses responsabilités comme il l'a fait tout au long de la pièce. Ainsi, au fil de ses rédactions, Claudio a réussi à créer un véritable conflit, non pas dans la fiction, mais dans l'esprit de son lecteur, Germán, qui est mis face à des choix éthiques, qu'il n'assume pas sous prétexte d'être professeur de Littérature et non pas de Morale ou de Religion (2014a: 430). Mais derrière Germán, il y a les autres lecteurs et spectateurs de Mayorga qui, comme lui, sont invités à entreprendre ce chemin d'apprentissage à l'envers, à questionner leurs certitudes et leur identité, à repenser leur conception de la civilisation et de la barbarie et à retracer la généalogie de la violence qu'ils portent potentiellement en eux. En ce sens, cette pièce illustre parfaitement ce «vrai» théâtre que Mayorga conçoit de la façon suivante - et nous clorons ces quelques réflexions sur cette citation:

El arte, dice Benjamin, no consiste en pulimentar, en dar brillo, sino en cepillar la realidad a contrapelo. El verdadero arte ha de ser duro, difícil, conflictivo, el conflicto más importante que ofrece el teatro no es aquel que se presenta en escena, sino aquel que se da entre el escenario y el patio de butacas. Un teatro que no divide al patio de butacas y que no divide al espectador es un teatro irrelevante. El verdadero arte ha de ser capaz de sembrar el conflicto en el corazón mismo del espectador. (2014b)

\section{Bibliographie}

Agamben G., 2006, Qu'est-ce qu'un dispositif, Paris, Payot et Rivages.

Benasayag M., 2015, Clinique du mal-être: la "psy» face aux nouvelles souffrances psychiques, Paris, Éditions La Découverte.

Bourdieu P., 1993, "À propos de la famille comme catégorie réalisée», Actes de la recherche en sciences sociales, 100 . 
Carnevali D., 2015, «La vida imaginada: El chico de la última fila de Juan Mayorga", in: Forma dramática y representación del mundo. Apuntes sobre la noción de fábula en el contexto teatral europeo contemporáneo, thèse doctorale soutenue à la Universitat Autónoma de Barcelona [inédite].

Comte A., 1853, Système de politique positiviste ou Traité de sociologie, instituant la religion de l'humanité, Paris, Carilian-Goeury.

Donat B., 2014, «La familia disfuncional es la protagonista del nuevo teatro », http://epoca1.valenciaplaza.com/ver/138251/familia-disfuncional-teatro. html [Consulté le 25/11/2016].

Durkheim E., 1963 (1897), Le suicide. Étude de sociologie, Paris, PUF.

Eid G., 2008, «La famille postmoderne: intimité et parentalité», La Revue de REDIF, 1.

Espeche M., 2014, «Sobre la psicología del ninguneo», La Nación (29 novembre).

Gap E., 2009, «Virtuose et vertigineux», Les Trois Coups - Le journal du spectacle vivant (13 mars).

Guerrand R.-H., 1991, «Le décor de l'intimité familiale. Approche historique», in: Singly F. (dir.), La famille. L'état des savoirs, Paris, Éditions de La Découverte.

Le Play F., 1884, La famille selon le vrai modèle signalé par l'histoire de toutes les races et de tous les temps, Tours, Alfred Mame et Fils Éditeurs.

Le Run J.-L., 2015, «Dynamique de séduction à l'adolescence: illustration littéraire et cinématographique», EnfanceßPsy, 4, p. 117-130.

Mayorga J., 2010, «Elipses de Benjamin», Constelaciones. Revista de Teoría Crítica.

Mayorga J., 2014a [2006], El chico de la última fila, in: Juan Mayorga. Teatro 1989-2014, Segovia, Ediciones La uÑa RoTa.

Mayorga J. 2014b, «La filosofía no es una disciplina académica, es un plan de vida; todos estamos llamados a ser filósofos", http://www.jotdown.es/2014/09/ juan-mayorga-la-filosofia-no-es-una-disciplina-academica-es-un-plan-devida-todos-estamos-llamados-a-ser-filosofos/ [Consulté le 25/11/2016].

Mayorga J., 2016, «Juan Mayorga: las obsesiones de un matemático y autor de éxito", El País (1 ${ }^{\text {er }}$ juin), http://cultura.elpais.com/cultura/2016/06/01/ actualidad/1464795827_334601.html [Consulté le 25/11/2016].

Pierron J.-P., 2014, Où va la famille?, Paris, Les Liens qui Libèrent.

Rodríguez Solás D., 2012, "La teatralización de la escritura en El chico de la última fila», Teatronarrativa-principal (9 novembre).

Sadowska Guillón I., "La mise en scène de l'écriture. Entretien avec Juan Mayorga», http://www.la-tempete.fr/spectacles_ressources/08_09/salle1_ legarcondudernierrang/entretien.pdf [Consulté le 25/11/2016].

Von Matt P., 1998, Fils dévoyés, filles fourvoyées. Les désastres familiaux dans la littérature, Paris, Éd. de la Maison des sciences de l'homme. 